\title{
Pre-Exposure Rabies Vaccination among US International Travelers: Findings from the Global TravEpiNet Consortium
}

\author{
Samantha B. Dolan,, Emily S. Jentes,, Mark J. Sotir,, Pauline Han, Jesse D. Blanton, ${ }^{1}$ Sowmya R. Rao, ${ }^{3,4}$ \\ Regina C. LaRocque, ${ }^{5,6}$ Edward T. Ryan ${ }^{5,6}$ and the Global TravEpiNet Consortium
}

\begin{abstract}
Background: People who travel to areas with high rabies endemicity and have animal contact are at increased risk for rabies exposure. We examined characteristics of international travelers queried regarding rabies vaccination during pretravel consultations at Global TravEpiNet (GTEN) practices during 2009-2010.

Material and Methods: We performed bivariate and multivariable analyses of data collected from 18 GTEN clinics. Travel destinations were classified by strength level of rabies vaccination recommendation.

Results: Of 13,235 travelers, 226 (2\%) reported previous rabies vaccination, and $406(3 \%)$ received rabies vaccine at the consultation. Common travel purposes for these 406 travelers were leisure (26\%), research/education $(17 \%)$, and nonmedical service work (14\%). Excluding the 226 who were previously vaccinated, $8070(62 \%)$ of 13,009 travelers intended to visit one or more countries with a strong recommendation for rabies vaccination; $1675(21 \%)$ of these 8070 intended to travel for 1 month or more. Among these 1675 travelers, $145(9 \%)$ were vaccinated, $498(30 \%)$ declined vaccination, $832(50 \%)$ had itineraries that clinicians determined did not indicate vaccination, and $200(12 \%)$ remained unvaccinated for other reasons. In both bivariate and multivariate analyses, travelers with trip durations $>6$ months versus $1-3$ months (adjusted odds ratio $[\mathrm{OR}]=4.9[95 \%$ confidence interval [CI] 2.1, 11.4]) and those traveling for "research/education" or to "provide medical care" (adjusted $\mathrm{OR}=5.1$ [95\% CI 1.9, 13.7] and 9.5 [95\% CI 2.2, 40.8], respectively), compared with leisure travelers, were more likely to receive rabies vaccination.

Conclusions: Few travelers at GTEN clinics received rabies vaccine, although many planned trips 1 month long or more to a strong-recommendation country. Clinicians often determined that vaccine was not indicated, and travelers often declined vaccine when it was offered. The decision to vaccinate should take into account the strength of the vaccine recommendation at the destination country, duration of stay, availability of postexposure prophylaxis, potential for exposure to animals, and likelihood of recurrent travel to high-risk destinations.
\end{abstract}

Key Words: Rabies vaccine-Travelers-Travel clinics—International destinations.

\section{Introduction}

$\mathbf{R}$ ABIES IS A VIRAL DISEASE THAT CAUSES an acute, almost always fatal encephalitis in humans; it is most commonly transmitted through saliva from a rabid animal bite. Approximately $99 \%$ of human rabies deaths occur in resource- limited countries, with an estimated 55,000 deaths annually in Africa and Asia (Knobel et al. 2005, Meslin 2005, World Health Organization 2005). Timely access to rabies postexposure prophylaxis (PEP), including thorough wound washing, administration of a series of vaccine doses, and for previously unvaccinated individuals, prompt infiltration with rabies

\footnotetext{
${ }^{1}$ Travelers' Health Branch, Division of Global Migration and Quarantine, Centers for Disease Control and Prevention, Atlanta, Georgia. ${ }^{2}$ Poxvirus and Rabies Branch, Division of High-Consequence Pathogens and Pathology, Centers for Disease Control and Prevention, Atlanta, Georgia.

${ }^{3}$ Department of Quantitative Health Sciences, University of Massachusetts Medical School, Worcester, Massachusetts.

${ }^{4}$ Center for Health Quality, Outcomes and Economic Research, Bedford VA Medical Center, Bedford, Massachusetts.

${ }^{5}$ Division of Infectious Diseases, Massachusetts General Hospital, Boston, Massachusetts.

${ }^{6}$ Harvard Medical School, Boston, Massachusetts.
} 
immune globulin (RIG) may be challenging in rural areas or areas with poor health care access and few resources.

An accurate rate of rabies exposures among travelers has not been calculated. However, a range of approximately 16200 possible exposures per 100,000 travelers has been reported (Centers for Disease Control and Prevention 2012a), and a recent study examining medical records following PEP estimated that $0.4 \%$ of travelers receive an at-risk animal bite per month of residence in a rabies-endemic country (Gautret and Parola 2012). A GeoSentinel study found that approximately $1.4 \%$ of travelers visiting medical clinics from 1998 to 2005 reported an animal-associated injury (Gautret et al. 2007). Furthermore, a 2008 study from Thailand assessed the risk of rabies exposure among 870 backpackers and found that $3.6 \%$ of them were licked and $0.7 \%$ were bitten by an animal (Piyaphanee et al. 2010).

Rabid dogs remain the major source of rabies virus transmission to humans, with more than $99 \%$ of human rabies deaths occurring after transmission from canids (World Health Organization 2005). In many cities in resource-limited countries, the primary risks to travelers are free-roaming dogs. Canine vaccination programs are nonexistent in many countries, including several in Africa and Asia, and as such, rabies is not well controlled in numerous areas of the world (Wunner and Briggs 2010). Travel to resource-limited countries increased from $31 \%$ of international tourist arrivals in 1990 to $47 \%$ in 2010 (UNWTO 2011). International travelers visiting these countries may experience an increased risk of exposure to rabies due to the higher prevalence of rabies in animals.

Unfortunately, many US travelers, including those visiting areas with high rabies endemicity, do not seek health advice before traveling (LaRocque et al. 2010), and therefore may not receive sufficient information about rabies. Even travelers who do seek pretravel health consultations might not understand the risk of rabies at their destinations, follow animal avoidance guidelines, or consider vaccination. To inform and improve pretravel health care recommendations regarding rabies, we sought to better understand the rabies vaccination status and travel itineraries of people seeking pretravel consultations at Global TravEpiNet (GTEN) practices.

\section{Materials and Methods}

GTEN is a consortium of US medical practices that is sponsored by the Centers for Disease Control and Prevention (CDC), as previously described (LaRocque et al. 2012b). GTEN sites are distributed across the United States and include academic practices, health care consortia, health maintenance organizations, pharmacy-based clinics, private practices, and public health clinics. GTEN was established in 2009 to collect data about international travelers seeking pretravel health care. For this investigation, we analyzed GTEN data collected from January 1, 2009, to December 31, 2010. During this period, GTEN consisted of 18 practices located in 12 US states. An institutional review board at each participating site reviewed and approved the study.

Data from all persons seen for pretravel consultations were collected using a secure online tool. During each consultation, travelers provided details about their medical history, itinerary, and travel purpose. Travelers were allowed to choose multiple purposes of travel, destination countries, and types of accommodation. For the purpose of this analysis, travelers who indicated that they were returning to the country of origin of self/family to visit friends and relatives were defined as visiting friends or relatives (VFR) travelers in accordance with the CDC definition (Centers for Disease Contol and Prevention 2012a), if they were visiting low- or low-middle income countries as defined by the World Bank's 2009 World Development Report (World Bank 2009).

Clinicians verified the information self-reported by travelers and entered additional data into the online tool regarding vaccines administered, medications prescribed, and health advice given at the consultation. For rabies vaccine, clinicians were prompted to provide a reason if the vaccine was not administered for those traveling $\geq 28$ days (defined as 1 month hereafter); possible reasons included having preexisting immunity, vaccine not indicated for this itinerary, referred to a primary care provider (PCP), medical contraindication, patient declined vaccine, insufficient time to complete vaccine series prior to departure, or vaccine not available. Rabies vaccine recipients were defined as travelers receiving a first dose of rabies vaccine at their consultation. Unvaccinated travelers were defined as those without preexisting immunity who were not vaccinated during the consultation. Previously vaccinated travelers self-reported that they had received a vaccination series before the GTEN consultation; however, no serologic tests were done to confirm immunity.

The strength level of the rabies vaccination recommendation for each country was classified by using an unpublished method from the CDC's Travelers' Health and Poxvirus and Rabies Branches. This method combines each country's canine rabies endemicity level with the availability of PEP. Four recommendation strength levels were used-weak, moderate, strong, or no recommendation listed (Appendix A). These vaccination recommendations are listed on each country destination page on the CDC's Travelers' Health website (www.cdc.gov/travel) (Centers for Disease Control and Prevention 2012b); the GTEN tool directly links to these countryspecific recommendations.

For travelers indicating more than one destination country, the country with the strongest recommendation strength was used to assess a traveler's risk of exposure to rabies during their trip. However, for our analysis of rabies vaccine status among long-term ( $\geq 1$ month) travelers, only travelers who reported a single destination country were included to accurately capture trip duration, because the GTEN tool did not collect the durations of stay for each country for travelers visiting multiple countries. Only travelers who reported one purpose of travel were included for comparisons between people traveling for different purposes.

All data were analyzed in SAS version 9.3 (SAS Institute, Cary, NC). We performed bivariate comparisons of traveler characteristics (e.g., means, medians, standard deviations) of vaccinated versus unvaccinated travelers. We used Wilcoxon rank sum tests to test for distribution differences between continuous variables. Chi-squared tests, odds ratios (OR), and $95 \%$ confidence intervals $(\mathrm{CI})$ were used for categorical variable comparisons. For bivariate and multivariable logistic regression analyses, we included only travelers who elected to receive rabies vaccination and those who declined it to identify predictors behind traveler decisions. Random intercept models using clinic site as the random effect and a correction 
for the small number of clusters (clinic sites) were used to account for possible between-clinic variation. The final multivariable model included purpose of travel (leisure, research/education, nonmedical service work, VFR, business, and providing medical care), duration of trip ( 1 to $\leq 3$ months, 4-6 months, and $>6$ months), age in years of travelers (children < 15 years, adults 15-49 years, and adults $>49$ years), and gender. Statistical significance was determined at a twosided 0.05 level for all tests.

\section{Results}

Of 13,235 travelers analyzed, $226(2 \%)$ reported previous vaccination, $406(3 \%)$ received rabies vaccine at their clinic visit, and 12,603 (95\%) remained unvaccinated against rabies. Travelers who received rabies vaccine were significantly younger than unvaccinated travelers (median 27 vs. 36 years, $p<0.01$ ) (Table 1). The most common purpose of travel for vaccine recipients was leisure $(26 \%)$; however, higher proportions of vaccinated travelers indicated research/education, nonmedical service work, adventure, providing medical care, and military service as a purpose of travel (Table 2). Although India was the most commonly visited country among all travelers, the countries with the highest proportions of travelers receiving rabies vaccine were Cambodia (8\%), Kenya (7\%), Vietnam (6\%), and Thailand (6\%) (Fig. 1).

Among the 13,009 travelers without a previous rabies vaccination, $8070(62 \%)$ planned to visit at least one country with a strong rabies vaccine recommendation. Of these 8070, 1675 (21\%) planned to travel for 1 month or more to only one country with a strong rabies vaccine recommendation. Clinicians determined that vaccination was not indicated for 832 $(50 \%)$ of these 1675 travelers (Table 3). Among the remaining 827 travelers for whom clinicians determined that vaccination was indicated (excluding those travelers with a medical contraindication or who were not queried for a vaccination status), 498 (60\%) declined rabies vaccine, 145 (18\%) received a

Table 1. Demographic Characteristics of Global TravEpiNet (GTEN) Rabies-VAccinated AND -Unvaccinated Travelers $(N=13,009), 2009-2010$

\begin{tabular}{lccc}
\hline & \multicolumn{3}{c}{ GTEN travelers $^{\mathrm{a}}$} \\
\cline { 2 - 4 } Characteristics & Vaccinated & Unvaccinated & All \\
\hline Total & 406 & 12603 & 13009 \\
Median & 27 & 36 & 35 \\
$\quad$ age (years) & & & \\
Age (years) & $n$ (column \%) & $n$ (column \%) & $n$ (column \%) \\
$<5$ & $23(6)$ & $441(4)$ & $464(4)$ \\
$6-17$ & $40(10)$ & $736(6)$ & $773(6)$ \\
$18-49$ & $288(71)$ & $7724(61)$ & $8012(62)$ \\
$50-64$ & $42(10)$ & $2594(21)$ & $2636(20)$ \\
$>64$ & $13(3)$ & $1108(9)$ & $1121(9)$ \\
Gender & & & \\
$\quad$ Male & $173(43)$ & $5803(46)$ & $5976(46)$ \\
Female & $233(57)$ & $6800(54)$ & $7033(54)$ \\
\hline
\end{tabular}

${ }^{a}$ Vaccinated travelers are those who received rabies vaccine at the GTEN consultation; unvaccinated travelers were defined as those without pre-existing immunity who were not vaccinated at the consultation.

${ }^{\mathrm{b}}$ Wilcoxon rank sum tests $p$ value $<0.0001$.

${ }^{\mathrm{c}}$ Chi-squared $p$ value $=0.1718$.
Table 2. Purpose of Travel for Global TravEpiNet (GTEN) RABIES-VACCINATED AND -UNVACCINATED Travelers $(N=16,039),(N$ [COLUMN \%] $)$

GTEN travelers ${ }^{\mathrm{b}}$

\begin{tabular}{|c|c|c|}
\hline Purpose of travel ${ }^{\mathrm{a}}$ & Vaccinated & Unvaccinated \\
\hline Leisure & $162(26)$ & $7762(50)$ \\
\hline Research/education & $110(17)$ & $1221(8)$ \\
\hline Nonmedical service work & $89(14)$ & $739(5)$ \\
\hline Business & $81(13)$ & $2085(13)$ \\
\hline Adventure & $74(12)$ & $738(5)$ \\
\hline Providing medical care & $57(9)$ & $554(4)$ \\
\hline VFR $^{\mathrm{c}}$ & $38(6)$ & $1350(9)$ \\
\hline Missionary work & $15(2)$ & $624(4)$ \\
\hline Attending large gathering & $3(1)$ & $260(2)$ \\
\hline Military service & $3(1)$ & $9(0)$ \\
\hline Adoption & 0 & $53(0)$ \\
\hline Receiving medical care & 0 & $12(0)$ \\
\hline Total purposes of travel chosen & 632 & 15,407 \\
\hline
\end{tabular}

aTravelers could choose more than one purpose for travel; therefore, the total purposes of travel exceed the number of vaccinated travelers $(n=406)$ and unvaccinated travelers $(n=12,603)$. The denominator is the total number of purposes of travel chosen $(n=16,039)$.

${ }^{b}$ Vaccinated travelers are those who received rabies vaccine at the GTEN consultation; unvaccinated travelers were defined as those without pre-existing immunity who were not vaccinated at the consultation.

${ }^{c}$ VFR indicates travelers participating in GTEN who selected "returning to country of origin of self or family to visit friends and relatives" and were visiting low- or low-middle income countries according to the 2009 World Bank World Development Report (available at http://econ.world bank.org), as defined previously by the CDC.

rabies vaccine, $130(16 \%)$ had insufficient time to receive an indicated rabies vaccine series, and 54 (7\%) remained unvaccinated for other reasons.

Among the 399 travelers included in the bivariate and multivariable analyses who declined a vaccination, 253 (63\%) were VFR travelers. In contrast, the most common purpose of travel for the 82 travelers receiving a vaccination at the consultation was research/education (33\%) (Table 4) Compared with leisure travelers, those traveling for research/education were nearly five times more likely to receive vaccination (adjusted OR $=5.1,95 \%$ CI 1.9, 13.7) and those traveling to provide medical care were more than nine times more likely to receive vaccination (adjusted $\mathrm{OR}=9.5,95 \% \mathrm{CI} 2.2,40.8$ ). Travelers going on trips longer than 6 months were significantly more likely to receive rabies vaccination (adjusted $\mathrm{OR}=4.9,95 \% \mathrm{CI} 2.1,11.4$ ) than those going on 1- to 3-month trips. For travelers going on trips 4-6 months long, the likelihood of receiving a rabies vaccination was not significant.

\section{Discussion}

Overall, a relatively small percentage of GTEN travelers received a pre-exposure rabies vaccination during their clinic visit, despite the fact that the majority were traveling to at least one country deemed by the CDC to have a strong recommendation for rabies vaccination. This finding is similar to those of previous studies that found that most travelers are not vaccinated for rabies before travel (Altmann et al. 2009, Gautret et al. 2008, Gautret et al. 2011, Piyaphanee et al. 2010). Specifically, $60 \%$ of GTEN travelers visiting a single strong- 


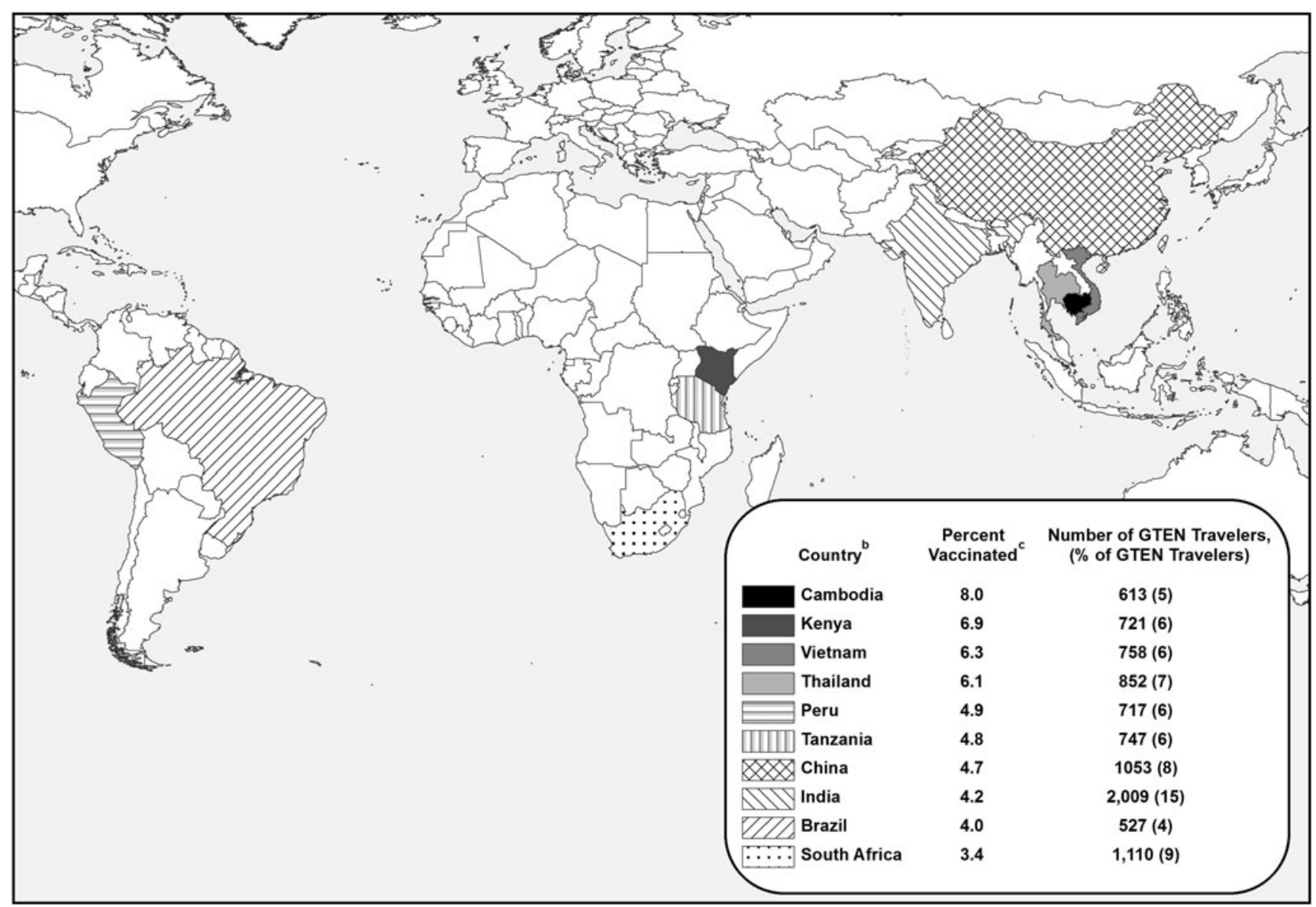

FIG. 1. Most common destinations for rabies-vaccinated travelers and the overall number of Global TravEpi Network (GTEN) travelers visiting those destinations $(n=13,009) .{ }^{\text {a }}$ Includes GTEN travelers who were not vaccinated prior to their clinic appointment. ${ }^{\mathrm{b}}$ Thailand has a moderate recommendation strength. The other nine countries have strong recommendations. Patients could choose multiple destinations for their itineraries. ${ }^{c}$ Vaccinated travelers are those who received rabies vaccine at the GTEN consultation; unvaccinated travelers were defined as those without pre-existing immunity who were not vaccinated at the consultation.

recommendation country for 1 month or more declined a recommended rabies vaccination. Other studies have found that travelers refused vaccination because of high cost, low perception of benefit, concern for an adverse reaction, and belief that vaccination was not necessary (Altmann et al. 2009, Piyaphanee et al. 2010, Gautret and Parola 2012). In fact, the average wholesale price of an individual rabies vaccine dose for pre-exposure vaccination can range from $\$ 194$ to $\$ 240$ (Anonymous 2008). However, these prices are likely an underestimate of what a traveler would pay because they do not take into account the direct price to the clinician, the clinician's administration and office visit fees, storage fees, or shipping costs.

Trip duration may also predict whether a traveler chooses to receive a rabies vaccine. We found that those traveling longer than 6 months were more likely to receive rabies vaccination than those going on 1- to 3-month-long trips. Travelers may view their risk of exposure as being dependent on and proportional to the duration of their trip. However, research has suggested that trip duration is not always predictive of a traveler's risk of rabies exposure (Gautret and Parola 2012). Although travelers staying for longer periods of time might encounter more potential rabies exposures, an indi- vidual may experience an exposure on the first day of a trip. Trip duration may not always predict a traveler's risk of rabies exposure; however, it should still be discussed as a potential risk factor during a consult, especially for those travelers with expected animal exposure (e.g., veterinarians, field biologists, animal handlers, cavers, missionaries), as well as for travelers going to areas where effective PEP might not be available (Centers for Disease Contorl and Prevention 2012a).

Most GTEN clinicians are travel medicine and vaccination specialists who regularly provide pretravel consultations to international travelers. In this analysis, GTEN clinicians deemed that rabies vaccine was not indicated for $50 \%$ of travelers, despite the fact that these travelers were going to a strong-recommendation country for more than 1 month. CDC recommendations do not specifically state that vaccine should be considered for all people traveling for more than a month to these areas; however, as mentioned, longer stays in rabiesendemic areas might increase rabies exposure risks (Centers for Disease Control and Prevention 2012a). The clinicians' reasons for deeming rabies vaccine as not indicated for travelers were not recorded for this study. The travelers' itineraries may have included activities and local destinations with 
Table 3. Rabies Vaccine Status among Global Travepinet (GTEN) Travelers Without Previous Rabies Vaccination Who Were Visiting One Strong-Recommendation Country, by Duration OF TRIP $(N=1675),(N$, [COLUMN \%])

\begin{tabular}{|c|c|c|c|c|c|}
\hline \multirow[b]{2}{*}{ Vaccine status } & \multicolumn{5}{|c|}{ Trip duration } \\
\hline & 1 to $\leq 3$ months & 3-6 months & $6-12$ months & $>1$ year & Total \\
\hline Administered at consultation & $74(6)$ & $22(13)$ & $37(26)$ & $12(18)$ & $145(9)$ \\
\hline Not indicated & $690(53)$ & $74(44)$ & $39(28)$ & $29(43)$ & $832(50)$ \\
\hline Referred to primary care provider & $6(1)$ & $1(1)$ & $1(1)$ & $1(2)$ & $9(1)$ \\
\hline Medical contraindication & $11(1)$ & 0 & 0 & 0 & $11(1)$ \\
\hline Patient declined & $386(30)$ & $46(27)$ & $48(34)$ & $18(27)$ & $498(30)$ \\
\hline $\begin{array}{l}\text { Insufficient time to complete vaccine series } \\
\text { prior to departure }\end{array}$ & $93(7)$ & $21(12)$ & $11(8)$ & $5(7)$ & $130(8)$ \\
\hline Vaccine not available & $34(3)$ & $4(2)$ & $4(3)$ & $3(4)$ & $45(3)$ \\
\hline Not queried $^{\mathrm{a}}$ & $4(0)$ & $1(1)$ & 0 & 0 & $5(0)$ \\
\hline Total & 1298 & 169 & 140 & 68 & 1675 \\
\hline
\end{tabular}

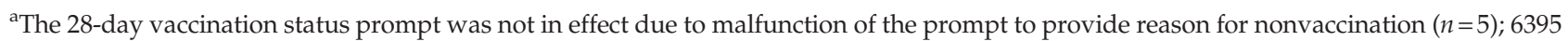
travelers had trip durations of 1-27 days.

lower risk of rabies exposure that the GTEN tool was unable to capture. GTEN clinicians would have received this information verbally and may have factored it into their vaccination recommendation. A range of factors is usually considered in deciding whether vaccines are indicated, including the destination, duration of travel, the likelihood of future travel or animal exposure, the purpose of travel, availability of PEP, and the possibility of medical evacuation to a location where PEP is available.
The timing of a traveler's pretravel consultation in relation to their departure date can impede a clinician's ability to provide a traveler with a complete rabies vaccination series. In this analysis, 130 travelers were unable to receive an indicated vaccine because time was insufficient to complete the vaccination series prior to their trip departure. CDC does not recommend that travelers initiate the rabies vaccine series if they have insufficient time to complete the series of vaccine, which is given on days 0,7 , and 21 or 28 days, prior to their

Table 4. Bivariate and Multivariable Models: Receiving Versus Declining a Rabies Vaccination among Global TravEpiNet (GTEN) Travelers with One Purpose of Travel Visiting One Strong-Recommendation Country for $\geq 28$ Days $(N=481)$

\begin{tabular}{|c|c|c|c|c|c|c|c|c|}
\hline \multirow[b]{3}{*}{ Characteristics } & \multirow{3}{*}{$\frac{\text { Received }(\mathrm{n}=82)}{\mathrm{n}(\operatorname{col} \%)}$} & \multirow{3}{*}{$\frac{\text { Declined }^{\mathrm{a}}(\mathrm{n}=399)}{\mathrm{n}(\operatorname{col} \%)}$} & \multicolumn{6}{|c|}{ Receiving vs. declining rabies vaccination } \\
\hline & & & \multicolumn{3}{|c|}{ Bivariate model } & \multicolumn{3}{|c|}{ Multivariable model } \\
\hline & & & OR & $95 \% C I$ & $\mathrm{p}$ value & OR & $95 \% C I$ & $\mathrm{p}$ value \\
\hline Purpose of travel & & & & & $<.01$ & & & $<.0001$ \\
\hline Leisure (referent) & $12(15)$ & $54(14)$ & 1.0 & - & - & 1.0 & - & \\
\hline Research/education & $27(33)$ & $47(12)$ & 5.5 & $2.3,13.4$ & & 5.1 & $1.9,13.7$ & \\
\hline Nonmedical service work & $8(10)$ & $14(4)$ & 2.5 & $0.9,6.4$ & & 2.4 & $0.8,7.0$ & \\
\hline VFR $^{\mathrm{b}}$ & $16(20)$ & $253(63)$ & 1.0 & $0.4,2.3$ & & 0.6 & $0.2,1.6$ & \\
\hline Business & $10(12)$ & $26(7)$ & 1.8 & $0.4,9.1$ & & 1.0 & $0.1,8.3$ & \\
\hline Providing medical care & $9(11)$ & $5(1)$ & 8.2 & $1.8,38.1$ & & 9.5 & $2.2,40.8$ & \\
\hline Trip duration length (months) & & & & & $<.01$ & & & $\leq 0.01$ \\
\hline 1-3 (referent) & $40(49)$ & $304(76)$ & 1.0 & - & - & 1.0 & - & \\
\hline $4-6$ & $14(17)$ & 42 (11) & 1.8 & $1.0,3.2$ & & 1.2 & $0.5,2.6$ & \\
\hline$>6$ & $28(34)$ & $53(13)$ & 4.7 & $3.0,7.4$ & & 4.9 & $2.1,11.4$ & \\
\hline Age (years) & & & & & 0.33 & & & 0.35 \\
\hline Adults, 15-49 (referent) & $52(63)$ & $200(50)$ & 1.0 & - & - & 1.0 & - & \\
\hline Children, $<15$ & $20(24)$ & $158(40)$ & 0.7 & $0.5,1.1$ & & 1.9 & $0.7,5.3$ & \\
\hline Older adults, $>49$ & $10(12)$ & $41(10)$ & 1.2 & $0.7,2.3$ & & 2.0 & $0.7,5.9$ & \\
\hline Gender & & & & & 0.31 & & & 0.50 \\
\hline Female (referent) & $50(39)$ & $205(51)$ & 1.0 & - & - & 1.0 & - & \\
\hline Male & $32(61)$ & $194(49)$ & 0.8 & $0.5,1.3$ & & 0.8 & $0.4,1.6$ & \\
\hline
\end{tabular}

aNinety-nine of the 498 travelers with trips 28 days or longer to one strong-recommendation country who declined a rabies vaccination were not included in the bivariate and multivariable analyses because information on the characteristics of these individuals was missing.

bVFR indicated travelers participating in GTEN who selected "returning to country of origin of self or family to visit friends and relatives" and were visiting low or low-middle income countries according to the 2009 World Bank World Development Report (Available at http:// econ.worldbank.org), as defined previously by the CDC.

$\mathrm{OR}$, odds ratio; CI, confidence interval. 
departure, because it may be problematic to plan PEP after a partial immunization series (Centers for Disease Control and Prevention 2012a). People receiving a rabies vaccine series are not considered preimmunized until the series is completed. Travelers who receive less than a full series of doses should be recommended to receive RIG and complete the vaccine series after a potential rabies exposure.

We also found that VFR travelers made up almost twothirds of those declining a vaccination. VFR travelers represent a unique risk group that makes up approximately $35 \%$ of international air travelers (Office of Travel and Tourism Industries 2011). Compared with other travelers, they have different travel activities and health care-seeking behaviors that may influence their decision to decline a vaccination (Hagmann et al. 2009). We found that $90 \%$ of VFR travelers planned to stay in homes with relatives, where risk of rabies exposure may differ from those lodging in a hotel. VFR travelers also typically travel for longer durations (Chen et al. 2009) and are more likely to visit only urban areas (Larocque et al. 2012a). In previous surveys, VFR travelers have shown a lower overall mean knowledge of rabies, compared with persons traveling for other purposes of travel (Angell et al. 2005, Altmann et al. 2009). Furthermore, a recent review of travel-related human rabies cases found that $43 \%$ of these travelers were VFRs (Carrara et al. 2013). Vaccination should be considered for those travelers, including VFRs, with a high likelihood of repeat travel or those moving to a rabiesendemic country (Cetners for Disease Control and Prevention 2012a).

Rabies education and access to PEP are crucial for travelers, because the consequences of not seeking timely and adequate care after an animal bite can be deadly. Recent deaths among US residents who were bitten and exposed to canine rabies overseas include a female traveler returning from Nepal (Basgoz 1998) and a US Army soldier returned from Afghanistan (Centers for Disease Control and Prevention 2012c). A pretravel health consultation, preferably 4-6 weeks before travel, is an opportunity for a health care provider to review a traveler's itinerary, along with his/her current health status, and provide health education and recommendations. Clinicians should use these consultations to educate travelers of appropriate actions in the event of an animal bite. It can be difficult for a traveler to receive PEP in areas with poor health care infrastructure, rural areas, or poor local transportation, thus travelers should consider the purchase of medical evacuation insurance. Clinicians should suggest that travelers review additional information concerning animal bite prevention and treatment through resources such as the CDC's Travelers' Health website (www.cdc.gov/travel) (Centers for Disease Control and Prevention 2012d).

Our study has several limitations. GTEN data might not be representative of all travelers or those seeking pretravel health consultations. Also, GTEN sites may differ in their decisions to recommend vaccination to travelers compared with other sites providing pretravel health consultations. The GTEN tool did not ask why travelers declined vaccination or why a clinician determined that vaccine was not indicated. The tool malfunctioned briefly and failed to query clinicians for the vaccination status of 5 travelers with trip durations 28 days or longer (Table 3). Another limitation was the use of the CDC's rabies country-specific recommendations. This categorization attempts to control for each country's canine rabies ende- micity and the availability of PEP; however, in the absence of scientific evidence, the categorization is made by expert opinion. This categorization may not accurately reflect an individual's actual risk, which is contingent on their specific activities, behaviors, and destinations within the country and these can vary in rabies endemicity. Finally, vaccine administration is affected by the supply of rabies vaccine, and clinics may not purchase or stock adequate quantities of vaccine.

\section{Conclusions}

This study highlights that rabies vaccine is administered to a low percentage of international travelers who may experience potential rabies exposures. Clinicians often determined that vaccine was not indicated, and travelers often declined vaccine when it was offered. Trip duration, trip activities, purpose of travel, and destination country should all be considered when recommending a rabies vaccine to a traveler. Although clinicians can strongly recommend a rabies vaccination to a traveler for a particular trip, obstacles such as the vaccine's cost and availability, lack of sufficient time to complete a vaccination series, and traveler misconceptions may prevent persons from receiving recommended vaccination. The decision to vaccinate should take into account the strength of the vaccine recommendation at the destination country, duration of stay, availability of PEP, potential for exposure to animals, and likelihood of recurrent travel to high-risk destinations.

\section{Acknowledgments}

The authors thank the travelers seen and the clinic staff at the GTEN clinics as of 2010. Members of the Global TravEpiNet Consortium include (in alphabetical order) George M. Abraham, Saint Vincent Hospital (Worcester, MA); Salvador Alvarez, Mayo Clinic (Jacksonville, FL); Vernon Ansdell and Johnnie A. Yates, Travel Medicine Clinic, Kaiser Permanente (Honolulu, HI); Elisha H. Atkins, Chelsea HealthCare Center (Chelsea, MA); John Cahill, Travel and Immunization Center, St. Luke's-Roosevelt (New York, NY); Holly K. Birich and Dagmar Vitek, Salt Lake Valley Health Department (Salt Lake, Utah); Bradley A. Connor, New York Center for Travel and Tropical Medicine, Cornell University (New York, NY); Roberta Dismukes and Phyllis Kozarsky, Emory TravelWell, Emory University (Atlanta, GA); Ronke Dosunmu, JourneyHealth (Maywood, NJ); Jeffrey A. Goad, International Travel Medicine Clinic, University of Southern California (Los Angeles, CA); Stefan Hagmann, Division of Pediatric Infectious Diseases, Bronx Lebanon Hospital Center (Bronx, NY); DeVon Hale, International Travel Clinic, University of Utah (Salt Lake City, UT); Noreen A. Hynes, John Hopkins Travel and Tropical Medicine, Division of Infectious Diseases, John Hopkins School of Medicine (Baltimore, MD); Frederique Jacquerioz and Susan McLellan, Tulane University (New Orleans, LA); Mark Knouse, Keystone Travel Medicine, Lehigh Valley Health Network (Allentown, PA); Jennifer Lee, Travel and Immunization Center, Northwestern Memorial Hospital (Chicago, IL); Regina C. LaRocque and Edward T. Ryan, Massachusetts General Hospital (Boston, MA); Alawode Oladele and Hanna Demeke, DeKalb County Board of Health Travel Services-DeKalb North and Central-T.O. Vinson Centers (Decatur, GA); Roger Pasinski and Amy E. Wheeler, Revere HealthCare Center (Revere, MA); Sowmya 
R. Rao, University of Massachusetts (Worcester, MA); Jessica Rosen, Infectious Diseases and Travel Medicine, Georgetown University (Washington, DC); Brian S. Schwartz, Travel Medicine and Immunization Clinic, University of California (San Francisco, CA); William Stauffer and Patricia Walker, HealthPartners Travel Medicine Clinics (St. Paul, Minnesota); and Joseph Vinetz, Travel Clinic, Division of Infectious Diseases, Department of Medicine, University of California-San Diego School of Medicine (La Jolla, CA). The authors also thank Gary Brunette, Ava Navin, and Clive Brown for their critical review of the manuscript, and Kevin Liske for his assistance in creating the figure.

Financial support for this article was received from the Centers for Disease Control and Prevention, Atlanta, GA (including U19CI000514 and U01CK000175).

\section{Author Diclosure Statement}

No competing financial interests exist.

The findings and conclusions in this report are those of the authors and do not necessarily represent the official position of the Centers for Disease Control and Prevention (CDC).

\section{References}

Altmann M, Parola P, Delmont J, Brouqui P, et al. Knowledge, attitudes, and practices of French travelers from Marseille regarding rabies risk and prevention. J Travel Med 2009; 16: 107-111.

Angell SY, Behrens RH. Risk assessment and disease prevention in travelers visiting friends and relatives. Infect Dis Clin North Am 2005; 19:49-65.

Anonymous. Red Book. Montvale, NJ: Thomson Healthcare, 2008; 2008.

Carrara P, Parola P, Brouqui P, Gautret P. Imported human rabies cases worldwide, 1990-2012. PLoS Negl Trop Dis 2013; 7:e2209.

Centers for Disease Control and Prevention. CDC Health Information for International Travel 2012. New York: Oxford University Press, 2012a.

Centers for Disease Control and Prevention. Destinations. Atlanta: Centers for Disease Control and Prevention, Traveler's Health Branch; 2012b (cited May 1, 2012). Available at http://wwwnc.cdc.gov/travel/destinations/list.htm

Centers for Disease Control and Prevention. Imported human rabies in a U.S. Army soldier-New York, 2011. Morbid Mortal Weekly Rep 2012c; 61:302-305.

Centers for Disease Control and Prevention. Travelers' Health. Atlanta: Centers for Disease Control and Prevention, Traveler's Health Branch; 2012d (cited September 1, 2012). Available at http://wwwnc.cdc.gov/travel/

Chen LH, Wilson ME, Davis X, Loutan L, et al. Illness in longterm travelers visiting GeoSentinel clinics. Emerg Infect Dis 2009; 15:1773-1782.

Gautret P, Parola P. Rabies vaccination for international travelers. Vaccine 2012; 30:126-133.

Gautret P, Schwartz E, Shaw M, Soula G, et al. Animal-associated injuries and related diseases among returned travellers: A review of the GeoSentinel Surveillance Network. Vaccine 2007; 30;25:2656-2663.
Gautret P, Shaw M, Gazin P, Soula G, et al. Rabies postexposure prophylaxis in returned injured travelers from France, Australia, and New Zealand: A retrospective study. J Travel Med 2008; 15:25-30.

Gautret P, Tantawichien T, Vu Hai V, Piyaphanee W. Determinants of pre-exposure rabies vaccination among foreign backpackers in Bangkok, Thailand. Vaccine 2011; 29:39313934.

Hagmann S, Benavides V, Neugebauer R, Purswani M. Travel health care for immigrant children visiting friends and relatives abroad: Retrospective analysis of a hospital-based travel health service in a US urban underserved area. J Travel Med 2009; 16:407-412.

Knobel DL, Cleaveland S, Coleman PG, Fevre EM, et al. Reevaluating the burden of rabies in Africa and Asia. Bull World Health Organ 2005; 83:360-368.

LaRocque RC, Rao SR, Tsibris A, Lawton T, et al. Pre-travel health advice-seeking behavior among US international travelers departing from Boston Logan International Airport. J Travel Med 2010; 17:387-391.

Larocque RC, Deshpande BR, Rao SR, Brunette GW, et al. Pretravel health care of immigrants returning home to visit friends and relatives. Am J Trop Med Hyg 2012a; 88:376380.

LaRocque RC, Rao SR, Lee J, Ansdell V, et al. Global TravEpiNet: A national consortium of clinics providing care to international travelers-analysis of demographic characteristics, travel destinations, and pretravel healthcare of high-risk US international travelers, 2009-2011. Clin Infect Dis 2012b; 54:455-462.

Meslin FX. Rabies as a traveler's risk, especially in high-endemicity areas. J Travel Med 2005; 12(Suppl 1):S30-S40.

Office of Travel and Tourism Industries. Profile of U.S. Resident Travelers Visiting Overseas Desintations: 2011 Outbound. 2011 (cited May 1, 2012). Available at http://tinet.ita.doc.gov/ outreachpages / download_data_table/2011_Outbound_ Profile.pdf

Piyaphanee W, Shantavasinkul P, Phumratanaprapin W, Udomchaisakul $\mathrm{P}$, et al. Rabies exposure risk among foreign backpackers in Southeast Asia. Am J Trop Med Hyg 2010; 82:1168-1171.

UNWTO. UNWTO Tourism Highlights, 2011 Edition. United Nations World Tourism Organization, 2011.

World Bank. World Development Report 2009: Reshaping economic geography. Washington DC: The World Bank, 2009.

World Health Organization. WHO Expert Consultation on Rabies. Contract no. 931. Geneva: WHO, 2005.

Wunner WH, Briggs DJ. Rabies in the 21 century. PLoS Neglect Trop Dis 2010; 4:e591.

Address correspondence to: Emily S. Jentes Travelers' Health Branch Division of Global Migration and Quarantine Centers for Disease Control and Prevention 1600 Clifton Road, MS E 03 Atlanta, GA 30333

E-mail: efj8@cdc.gov 


\section{Appendix A}

Rabies Vaccination Recommendation Strengths Listed on Country Pages of cdc.gov/travel ${ }^{\mathrm{a}}$

No recommendation

Country page shows no information on rabies vaccinations.

Weak recommendation

Rabies vaccination is recommended only for travelers involved in any activities that might bring them into direct contact with bats. These travelers include wildlife professionals, researchers, veterinarians, or adventure travelers visiting areas where bats are commonly found.

Moderate recommendation

Rabies vaccination is recommended only for travelers with significant occupational risks, such as veterinarians, and for long-term travelers and expatriates living in areas with a significant risk of exposure. Travelers involved in any activities that might bring them into direct contact with bats, carnivores, and other mammals, such as wildlife professionals, researchers, veterinarians, or adventure travelers visiting areas where bats, carnivores, and other mammals are commonly found.

Strong recommendation

Recommended for travelers spending a lot of time outdoors, especially in rural areas, involved in activities such as bicycling, camping, or hiking. Also recommended for travelers with significant occupational risks, such as veterinarians, and for long-term travelers and expatriates living in areas with a significant risk of exposure. Children are considered at higher risk because they tend to play with animals, may receive more severe bites, or may not report bites.

${ }^{a}$ Based on unpublished methodology for assigning country-specific recommendations for rabies. 